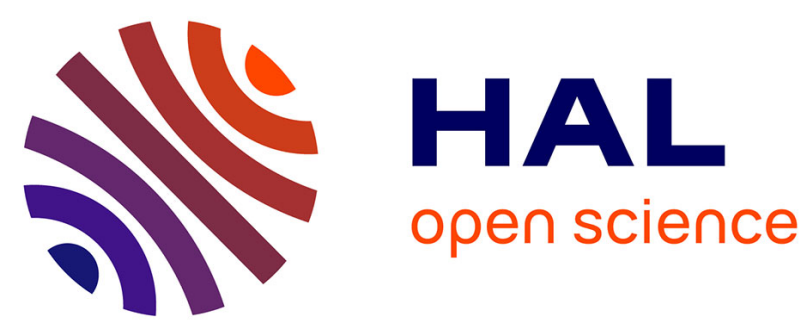

\title{
Impact of Small Cells Location on Performance and QoS of Heterogeneous Cellular Networks
}

\author{
Jean-Marc Kélif, Stéphane Senecal, Marceau Coupechoux
}

\section{To cite this version:}

Jean-Marc Kélif, Stéphane Senecal, Marceau Coupechoux. Impact of Small Cells Location on Performance and QoS of Heterogeneous Cellular Networks. IEEE International Symposium on Personal, Indoor and Mobile Radiocommunications (PIMRC), Sep 2013, London, United Kingdom. pp.1-6. hal-01220104

\section{HAL Id: hal-01220104 https://hal-imt.archives-ouvertes.fr/hal-01220104}

Submitted on 24 Oct 2015

HAL is a multi-disciplinary open access archive for the deposit and dissemination of scientific research documents, whether they are published or not. The documents may come from teaching and research institutions in France or abroad, or from public or private research centers.
L'archive ouverte pluridisciplinaire $\mathbf{H A L}$, est destinée au dépôt et à la diffusion de documents scientifiques de niveau recherche, publiés ou non, émanant des établissements d'enseignement et de recherche français ou étrangers, des laboratoires publics ou privés. 


\section{Impact of Small Cells Location on Performance and QoS of Heterogeneous Cellular Networks}

\author{
Jean-Marc Kelif and Stephane Senecal \\ Orange Labs \\ 38-40 rue du Général Leclerc, 92130 Issy-Les-Moulineaux, France \\ \{jeanmarc.kelif, stephane.senecal\}@orange.com
}

\author{
Marceau Coupechoux \\ Telecom ParisTech and CNRS LTCI \\ 23 avenue d'Italie, 75013 Paris, France \\ marceau.coupechoux@ telecom-paristech.fr
}

\begin{abstract}
We propose an analysis of the impact of the deployment of small base stations in a wireless network constituted of macro base stations. This analysis is particularly focused on the influence of the position and the transmitting power of small base stations on the performance of the network. In this aim, we consider an analytical model for heterogeneous cellular networks, composed of macro cells and small cells. The network model framework developed allows to derive closed form formulas for the Signal to Interference plus Noise Ratio (SINR) received by a mobile, whatever its location. Moreover, the proposed analytical model is validated by numerical simulations and it is shown that it is a good approximation of the SINR. Performance and quality of service (QoS) in terms of throughput and coverage can therefore be analyzed in a simple way. It makes it possible to analyze the deployment of small cells in an existing macro cells network.
\end{abstract}

Keywords-heterogeneous networks, small cells, location, performance, quality of service, throughput and coverage analysis

\section{INTRODUCTION}

One of the great challenges for wireless telecommunication operators is to answer to the exploding traffic demand, and particularly to deploy high data rates services. For the outdoor situations, a new solution known as Small Cell Networks (SCN) recently came out. Indeed, by increasing the number of cells in a given zone, a higher number of users may theoretically be served. However, a scalability problem appears: when the number of cells in a given zone increases, interference increases as well and may become a bottleneck for the performance. Moreover, when the density of transmitters increases, the increasing density of user equipments (UE) do not allow to cope with interference by using techniques based on the reuse of frequency or on spatial diversity [1]. A major limitation for the use of SCN is thus the intercell interference from other small cells or from macro cells, which can be of significant amount and thus renders the network unoperable. Another interest of SCN consists in the possibility to increase the coverage and the capacity of a wireless macro cell network. This paper presents an analytical evaluation of the impact of the deployment of small cells on a network composed of macro cells. We particularly focus on performance and quality of service (QoS) in terms of throughput and coverage. Moreover, we analyze the impact of the transmitting power of small base stations. An important issue is linked to the interference induced by adding small base stations in an existing network of macro cells. Indeed, the interferences induced may have an impact on the throughput and the coverage. This requires the analysis of the Signal to Interference plus Noise Ratio (SINR). The fluid model we propose in this paper is an analytical approach which allows to easily determine SINR and performance, specifically for heterogeneous networks. Recently, articles [2], [3] and [4] proposed an analysis of wireless networks with small cells deployment. In reference [2], the impact of reducing the cell size on the energy performance is studied. In reference [3], a small cell network is analyzed with cooperative power control. It shows that the system performance can be improved. In reference [4], authors propose an analysis of the channel allocation in a network composed of small cells and macro cells. The impact of decreasing the transmitting power of the macro base stations is addressed in [5], when femto base stations are deployed in an indoor environment. In [5], femto base stations serve only indoor users, and macro base stations serve only outdoor users. As a consequence, femto base stations have not the possibility to serve outdoor users even if macro base stations are overloaded. Also, recent references concerning heterogeneous mobile communication networks deployment mainly address the problems and challenges of intercell interference coordination and management techniques, as shown in [6] and [7] for instance.

The work presented in this paper deals specifically with the problem of performance and QoS estimation for heterogeneous cellular networks. This paper focuses on the SINR and throughput achievable in a given zone, in an outdoor environment composed of both small and macro cells. The users are connected to the best server base station, i.e. the one which allows the user to receive the best SINR. We first focus our study on the critical impact of the location of small base stations on the performance of the network. We then analyze the impact of the small base stations transmit power on the SINR, and the achieved throughput and coverage. In this paper, we develop an analytical fluid model for heterogeneous wireless networks, i.e. composed of two types of base stations: macro base stations (MBS) and small base stations (SBS). This model is partly based on the analytical fluid model for wireless networks proposed and validated in [8], and also through comparisons with a simulation tool developed and 
used at France Telecom/Orange. This model was originally developed for homogeneous networks, i.e. composed of only one type of base stations. We focus on the performance for a heterogeneous cellular network in terms of throughput, considering a LTE system with a $10 \mathrm{MHz}$ bandwidth (see [9] and [10] for more details).

The paper is thus organized as follows. In section II, we present the system model. We particularly establish the expression of the SINR via the analytical fluid model in the case of a heterogeneous network composed of macro base stations and small base stations. In section III, the scenarios and the results are described. This section analyzes the impact of the transmitting power on the throughput achieved by a mobile connected to its serving base station, when interference is induced by both small cells and macro cells. Section IV concludes the paper.

\section{SYSTEM MODEL}

We consider a wireless network composed of $M$ macro cells, $S$ small cells and $U$ user equipments. We focus our analysis on the downlink, in the context of an OFDMA based wireless network (e.g. WiMax, LTE).

Let us consider:

- $\mathcal{M}=\{1, \ldots, M\}$ the set of MBS, uniformly and regularly distributed over the two-dimensional plane.

- $\mathcal{S}=\{1, \ldots, S\}$ the set of SBS, uniformly and regularly distributed over the two-dimensional plane.

- $J$ sub-bands $j \in \mathcal{J}=\{1, \ldots, J\}$ where we denote $W$ the bandwidth of each sub-band.

- Each sub-band consists in a fixed number $s$ of subcarriers.

- $P_{i j}^{(k)}(u)$ the transmitted power assigned by base station $k$ to sub-carrier $i$ in sub-band $j$ towards user $u$.

- $g_{i j}^{(k)}(u)$ the propagation gain between transmitter $k$ and user $u$ in sub-carrier $i$ in sub-band $j$.

We assume that time is divided into slots. Each slot consists in a given sequence of OFDMA symbols. Since the time is slotted, transmissions within each cell do not interfere one with each other. We assume that there is no interference between sub-carriers. The total amount of power received by an UE u connected to a BS $k_{0}$, on sub-carrier $i$ of sub-channel $j$ is given by the sum of : useful signal $P_{i j}^{\left(k_{0}\right)}(u) g_{i j}^{\left(k_{0}\right)}(u)$, interference due to the other transmitters $\sum_{k \in \mathcal{S} \cup \mathcal{M}, k \neq k_{0}} P_{i j}^{(k)}(u) g_{i j}^{k}(u)$ and thermal noise $N_{t h}$.

We consider the SINR $\gamma_{i j}(u)$ defined by:

$$
\gamma_{i j}(u)=\frac{P_{i j}^{\left(k_{0}\right)}(u) g_{i j}^{\left(k_{0}\right)}(u)}{\sum_{k \in \mathcal{S} \cup \mathcal{M}, k \neq k_{0}} P_{i j}^{(k)}(u) g_{i j}^{k}(u)+N_{t h}}
$$

as the criterion of radio quality. To compute the total interfering power received by an UE, we consider the fluid model network approach [8], and extend it to networks composed of MBS and SBS. As we investigate the performance issue of heterogeneous network, we consider a worst case scenario where all the subcarriers are allocated to UEs. Consequently, each sub-carrier $i$ of the sub-band $j$ of any station is used and interfers with the ones of other stations.

\section{A. Analytical fluid model for homogeneous networks}

The fluid model core idea consists in replacing a given fixed finite number of transmitters by an equivalent continuous density of transmitters. A similar concept was proposed in references [11] and [12]. These works however do not provide a simple analytical formula for the SINR and focus only on massively dense networks. Our approach deals with general networks where the inter-nodes distance may be high as it is the case for realistic mobile communication networks. The network considered is characterized by a density of interfering base stations $\rho_{B}$, i.e. which use the same frequency bandwidth. The fluid model network approach, cf. [8] and [13], gives a closed-form formula of the interference factor ${ }^{1}$ as a function of the location of an UE. We remind hereafter the main results of this model as described in [8] and [13] (this last reference includes an extension to OFDMA). Let us consider a path gain $g_{t, u}=K r^{-\eta}$, where $K$ is a constant, $P_{t}$ the transmitted power, $r$ is the distance between a transmitter $t$ and a receiver $u$, and $\eta>2$ is the path loss exponent. For an UE at a distance $r$ from its serving base station, we can express the sum of interfering powers $I(r)$ as:

$$
I(r)=\frac{2 \pi P_{t} K \rho_{B}}{\eta-2}\left(2 R_{c}-r\right)^{2-\eta}
$$

where $2 R_{c}$ is the inter-site distance between BS. Formula (2) holds for all distance values between BS, and remains valid even when this distance reaches several kilometers.

\section{B. Fluid model for heterogeneous networks}

We now consider a network composed of macro cells and small cells. This network is not homogeneous since the joint distribution of macro and small cells is not homogeneous. Moreover, the transmitting powers of MBS are different than the transmitting powers of SBS. The basic idea of the model is to consider that the network is composed of two types of transmitters, each one being spatially distributed according to a given distribution, which share the same bandwidth: a spatial distribution of $M$ macro cells of mean radius $R_{m}$ and a spatial distribution of $S$ small cells of mean radius $R_{s}$.

Let consider $P_{i j}^{(k)}$ as a constant for all $i, j, k$ for each type of transmitter, denoted $P_{s}$ for a SBS and $P_{m}$ for a MBS. We can drop the sub-indices and analyze the interference between sub-carriers.

1) UE connected to a MBS: We can express (1), for an UE connected to a macro base station denoted $m_{0}\left(i . e k_{0}=m_{0}\right)$ as:

$$
\frac{P_{m}(u) g_{i j}^{\left(m_{0}\right)}(u)}{\sum_{m \in \mathcal{M}, m \neq m_{0}} P_{m}(u) g_{i j}^{(m)}(u)+\sum_{s \in \mathcal{S}} P_{s}(u) g_{i j}^{(s)}(u)+N_{t h}}
$$

\footnotetext{
${ }^{1}$ The interference factor is defined as the ratio between the power coming from the serving BS and the sum of other BS powers received by an UE, considering all BS transmit with power $P_{t}$.
} 
Without any loss of generality, we consider that the SBS $s_{0}$ is located inside the coverage zone of the MBS denoted $m_{0}$ (see figure 1). We can rewrite (3), using the path gain $g_{s, u}=$ $K_{s} r^{-\eta}$ (for SBS) and $g_{m, u}=K_{m} r^{-\eta}$ (for MBS) as:

$$
\gamma\left(r_{m_{0}}\right)=\frac{r_{m_{0}}^{-\eta}}{\sum_{m \neq m_{0}} r_{m}^{-\eta}+\frac{K_{s} P_{s}}{K_{m} P_{m}}\left(r_{s_{0}}^{-\eta}+\sum_{s \neq s_{0}} r_{s}^{-\eta}\right)+\frac{N_{t h}}{K_{m} P_{m}}}
$$

where $r_{k}$ is the distance between the UE and the transmitter $k$. Using the fluid model, we consider a density $\rho_{s}$ of SBS and $\rho_{m}$ of MBS. The mean distance between SBS (resp. MBS) is $2 R_{s}$ (resp. $2 R_{m}$ ). Denoting $K=\frac{K_{s} P_{s}}{K_{m} P_{m}}$, we can express the SINR (4) as:

$$
\gamma\left(r_{m_{0}}\right)=\frac{r_{m_{0}}^{-\eta}}{I_{s}^{m_{0}}+I_{m}^{m_{0}}+\frac{N_{t h}}{K_{m} P_{m}}}
$$

where

$$
\begin{aligned}
I_{m}^{m_{0}} & =\frac{2 \pi \rho_{m}}{\eta-2}\left(2 R_{m}-r_{m_{0}}\right)^{2-\eta} \\
I_{s}^{m_{0}} & =K \frac{2 \pi \rho_{s}}{\eta-2}\left(2 R_{s}-r_{s_{0}}\right)^{2-\eta}+K r_{s_{0}}^{-\eta}
\end{aligned}
$$

We denote $\gamma^{0}\left(r_{s_{0}}\right)$ the SINR of the UE connected to a SBS when there is no MBS and $\gamma^{0}\left(r_{m_{0}}\right)$ the SINR of the UE connected to a MBS when there is no SBS. Considering that the thermal noise $N_{t h}$ is negligible, these SINRs can be expressed as (using fluid model, cf. [8]):

$$
\begin{aligned}
\gamma^{0}\left(r_{s_{0}}\right) & =\frac{(\eta-2) r_{s_{0}}^{-\eta}}{2 \pi \rho_{s}\left(2 R_{s}-r_{s_{0}}\right)^{2-\eta}} \\
\gamma^{0}\left(r_{m_{0}}\right) & =\frac{(\eta-2) r_{m_{0}}^{-\eta}}{2 \pi \rho_{m}\left(2 R_{m}-r_{m_{0}}\right)^{2-\eta}}
\end{aligned}
$$

Using these expressions, we can rewrite (5) as:

$$
\gamma\left(r_{m_{0}}\right)=\frac{\gamma^{0}\left(r_{m_{0}}\right)}{1+\Omega_{m}\left(1+\gamma^{0}\left(r_{s_{0}}\right)\right)}
$$

where

$$
\Omega_{m}=\frac{K_{s} P_{s}}{K_{m} P_{m}} \cdot \frac{\rho_{s}}{\rho_{m}} \cdot \frac{\left(2 R_{s}-r_{s_{0}}\right)^{2-\eta}}{\left(2 R_{m}-r_{m_{0}}\right)^{2-\eta}}
$$

2) UE connected to a $S B S$ : Following a similar way, we express (1), for an UE connected to a SBS denoted $s_{0}$ (i.e $\left.k_{0}=s_{0}\right)$ as:

$$
\begin{aligned}
\gamma\left(r_{s_{0}}\right) & =\frac{r_{s_{0}}^{-\eta}}{I_{s}^{s_{0}}+I_{m}^{s_{0}}+\frac{N_{t h}}{K_{s} P_{s}}} \\
I_{m}^{s_{0}} & =\frac{1}{K} \frac{2 \pi \rho_{m}}{\eta-2}\left(2 R_{m}-r_{m_{0}}\right)^{2-\eta}+\frac{1}{K} r_{m_{0}}^{-\eta} \\
I_{s}^{s_{0}} & =\frac{2 \pi \rho_{s}}{\eta-2}\left(2 R_{s}-r_{s_{0}}\right)^{2-\eta}
\end{aligned}
$$

In a similar way, we can express (12) as:

$$
\gamma\left(r_{s_{0}}\right)=\frac{\gamma^{0}\left(r_{s_{0}}\right)}{1+\Omega_{s}\left(1+\gamma^{0}\left(r_{m_{0}}\right)\right)}
$$

using (8) and (9) where

$$
\Omega_{s}=\frac{K_{m} P_{m}}{K_{s} P_{s}} \cdot \frac{\rho_{m}}{\rho_{s}} \cdot \frac{\left(2 R_{m}-r_{m_{0}}\right)^{2-\eta}}{\left(2 R_{s}-r_{s_{0}}\right)^{2-\eta}}
$$

3) Interest of the fluid model formula: The classical way to compute SINR is to consider the distances between the UE and each bas station (MBS and SBS). This approach results in an untractable formula which we need to approximate or to simulate. Our solution reduces the computational burden and also comes up with simple and interpretable analytical formulas (10) (15).

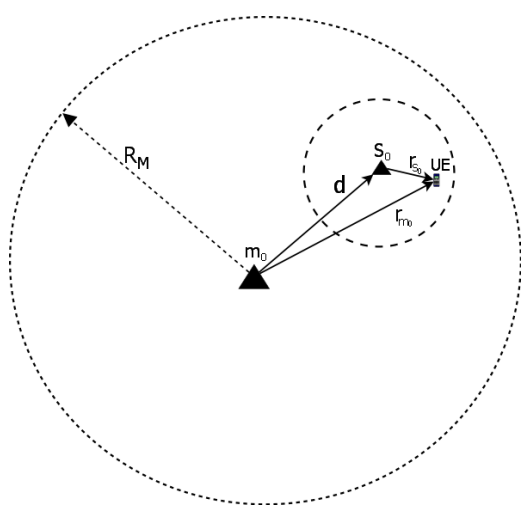

Fig. 1. Model of small-macro location.

4) Heterogeneity of the network: The closed form formula (10) and (15) of the SINRs are very interesting for many reasons. Indeed, they show that to calculate the SINR of an UE in a heterogeneous network, we can in a first step consider independently the SINR in a "sub-network" only composed of MBS and the SINR in a "sub-network" only composed of SBS. In a second step, we can consider the term $\Omega_{m}$ (or $\Omega_{s}$ ) which characterizes the impact of heterogeneity of the network. This term expresses that this impact depends on the characteristics of the system: the ratio of the density of each type of BS and their transmitting powers, the propagation parameters, the relative location of the UE to each type of transmitters.

In the case of an UE connected to a SBS (resp. MBS), we notice that when $\Omega_{s} \rightarrow 0$ (resp. $\Omega_{m} \rightarrow 0$ ), $\gamma\left(r_{s_{0}}\right) \rightarrow \gamma^{0}\left(r_{s_{0}}\right)$ (resp. $\left.\gamma\left(r_{m_{0}}\right) \rightarrow \gamma^{0}\left(r_{m_{0}}\right)\right)$. This case may happen for different configurations, for example when the density $\rho_{s}$ of SBS becomes very high compared to $\rho_{m}$, or when the ratio of the transmitting powers of MBS and SBS $\frac{P_{m}}{P_{s}}$ is very low. It means that the impact of the MBS becomes negligible, as if the network was only composed of SBS. Moreover, this term allows to quantify the relative impact of MBS and SBS on the SINR.

Expressions (10) and (15) are also useful to perform an analysis of heterogeneous small-macro cell networks. These expressions represent non-trivial extensions of formula derived for a standard homogeneous network. Indeed, these analytical expressions allow to calculate in a simple way the SINR of a mobile connected to a SBS whatever its location is. They particularly enable an analysis of the impact of transmitting SBS power on performance and QoS of UEs connected to SBS.

5) Impact of SBS location: The distances $r_{s_{0}}$ and $r_{m_{0}}$ can be expressed according to the distance $d$ between macro and small base stations (see figure 1): $r_{m_{0}}^{2}=r_{s_{0}}^{2}+d^{2}-2 d r \cos \theta$, 
where $\theta$ is the angle between $\vec{d}$ and $\vec{r}_{m_{0}}$. Therefore, this expression makes it possible to analyze the impact of the distance between SBS and MBS.

Remark: Let us notice that the analysis which allows to establish expressions (10) and (15) focuses on OFDMA based systems, such as WiMax and LTE, is still available for CDMA or HSPA systems.

\section{Validation of the fluid model for heterogeneous networks}

In this section, we propose a validation of the fluid model for the heterogeneous network presented in the last section. In this perspective, we will compare the cumulative density function (CDF) of the SINR obtained by using fluid expressions established in section II-B to those obtained numerically by simulations. Our simulator assumes a hexagonal network constituted of macro base stations. There are several rings of cells surrounding a central cell. Moreover, a small base station is located in each macro cell. The distance between the SBS and the MBS is $0.7 R_{m}$, the transmitting power are respectiveley $26 \mathrm{dBm}$ (SBS) and $43 \mathrm{dBm}$ (MBS). We assume a uniform distribution of UE. Figure 2 shows that the CDF calculated by using the fluid model for heterogeneous networks are very closed to the ones obtained by simulations.
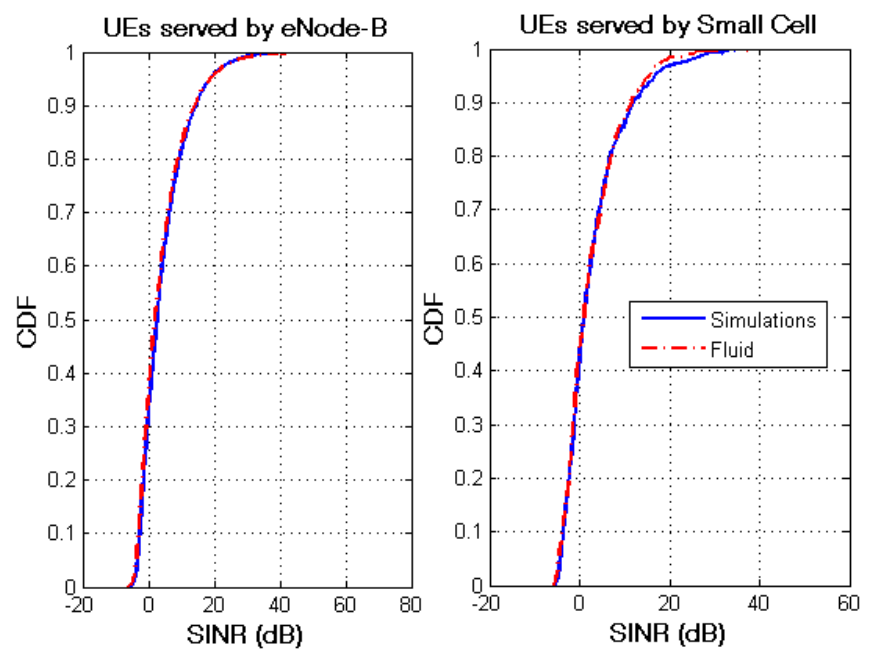

Fig. 2. CDF of the SINR for a UE connected to a MBS (left) and to a small cell (right) $d=0.7 R_{m}$.

\section{Throughput calculation}

The reachable throughput $D_{u}$ of an UE $u$ can be calculated from Shannon expression. Considering a bandwidth $W$, we have:

$$
D_{u}=W \log _{2}\left(1+\gamma_{u}\right)
$$

We can thus write the throughput of an UE connected to a MBS, as (using (10)):

$$
D\left(r_{m_{0}}\right)=W \log _{2}\left(1+\frac{\gamma^{0}\left(r_{m_{0}}\right)}{1+\Omega_{m}\left(1+\gamma^{0}\left(r_{s_{0}}\right)\right)}\right)
$$

and, in a similar way, we can write the throughput of an UE connected to a SBS, as (using (15)):

$$
D\left(r_{s_{0}}\right)=W \log _{2}\left(1+\frac{\gamma^{0}\left(r_{s_{0}}\right)}{1+\Omega_{s}\left(1+\gamma^{0}\left(r_{m_{0}}\right)\right)}\right)
$$

Expressions (18) and (19) enable to calculate the theoretical maximum achievable throughputs.

Remarks: Let notice that the mapping between the received SINR and the achieved throughput are established by the mean of link curves in the case of realistic network systems.

The network fluid model was validated by comparisons with curves generated by 3GPP system simulator for both the case of homogeneous network and the specific case of an heterogeneous network.

\section{SCEnARIO AND RESUlTS}

We present the results obtained from the heterogeneous fluid model applied to the analysis of a macro cell network in which small cells are added. We focus on a scenario where the macro cells and small cells share the same bandwidth. Therefore, a macro cell is interfered by small cells and by the other macro cells. Our aim is to determine the interest of adding small cells in an existing MBS network, in terms of performance (SINR and throughput) and coverage, when they share the same frequency band as the macro cell network. We also analyze the impact of transmitting power and deployment of SBS. The MBS locations and features are fixed and represent constraints for the problem addressed in the paper.

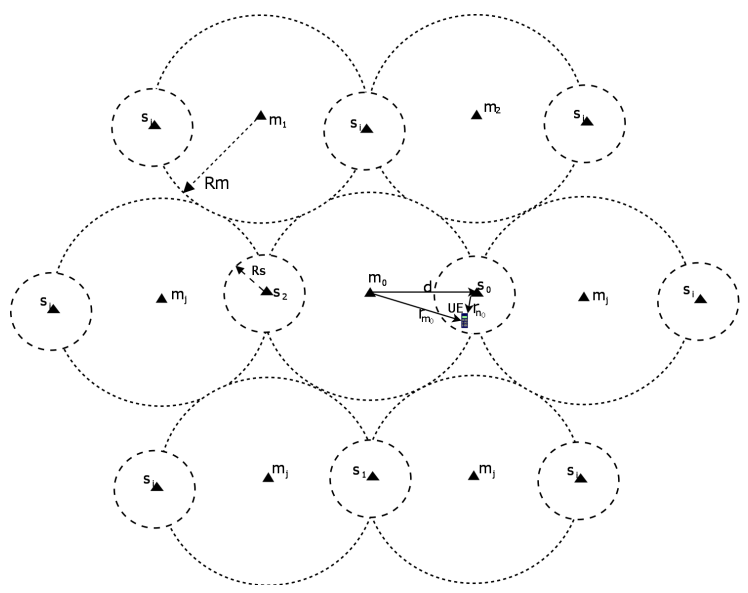

Fig. 3. Instance of scenario deployment for $d=R_{m}$.

We aim to determine the performance in terms of throughput reached by an UE connected to the best server.

\section{A. Assumptions}

Various parameters may have an impact on the SINR, the throughput and the coverage of BSs. We present hereinafter the parameters we chose in our analysis:

- downlink OFDM LTE, carrier frequency $2.6 \mathrm{GHz}$, channel bandwidth $10 \mathrm{MHz}$, 
- the SBS transmitting power: we set the value of $31 \mathrm{dBm}$, for most of the experiments,

- the MBS transmitting power: we set it at $43 \mathrm{dBm}$, as in a realistic transmission environment,

- the pathloss parameter : $\eta=3.5$,

- the mean distance $d$ between MBS and SBS,

- the location of the UE from its serving base station.

To illustrate the previously mentioned approach, we consider a heterogeneous network composed of macro cells and small cells. Macro cells are homogeneously placed on the network area. Small cells too. Different cases are considered corresponding to different mean distances $d$ between MBS and SBS. The figure 3 depicts the specific case where small base stations are located at the edge of macro cells (i.e. $d=R_{m}$ ). The mean distance between two MBS $2 R_{m}$ is equal to the mean distance between two SBS $2 R_{s}$ (1000 meters). SBS are added in the macro cell network in the aim to increase the performance (in terms of throughput and capacity) and the coverage of the system. Therefore, we consider that a mobile is connected to the $\mathrm{BS}$ which offers the best signal. This is a classical connection criterion of a mobile to its serving $\mathrm{BS}$, for wireless networks. The cumulative density functions (CDF) of the SINR and throughput characterize the outage probability and the performance distribution. Therefore, it is interesting to establish these CDF curves to analyze the QoS and the performance of the system. Indeed, these analyses allow to guarantee these QoS and performance results for various application types run under the network.

\section{B. Impact of the SBS location on the SINR distribution}

Firstly, we analyze the distribution of the best SINR received by an UE. At each location of the zone covered by a MBS, the SINR received from the MBS is compared to the one received from the SBS, and the station with the best SINR is chosen. The best SINR is calculated according to expression (10) or (15). Figure 4 depicts the cumulative density function (CDF) of the best SINR offered to the UE, for different distances $d$ from the MBS, and without small cells. These curves show that when the small cells are deployed at a distance $d=0.7 R_{m}$ or $d=R_{m}$, the CDF of the SINR is improved compared to the case without SBS. However, when SBS are located at distance $d=0.3 R_{m}$, the CDF of the SINR is slighly deteriorated. Therefore, since the coverage of a BS is an increasing function of the SINR, adding SBS does not always improve the coverage of a BS. For the same reason, the reachable QoS, in terms of throughput and outage probability, may also increase or decrease by adding a SBS, according to its location. These results can be explained as follows. A small cell allows to locally improve the SINR of an UE. However, it also induces more interferences on macro cells signals received by UEs (see expression (4)). To understand this phenomenon, let us consider an UE connected to a MBS. When SBS are too close to MBS (in figure 4, this case happens for $d=0.3 R_{m}$ ), these interferences have a relatively high level and, as a consequence, an UE connected to a macro BS has a lower SINR than without SBS. When SBS are far enough from MBS (in figure 4, this case happens for $d=0.7 R_{m}$ ), these interferences are relatively low and do not have a high impact on the SINR of the UE. For instance, from figure 4 we can deduce that the presence of a small cell (SC) at the edge of the zone covered by a MBS increases the number of mobiles which reach a SINR of $10 \mathrm{~dB}$ by $25 \%$ (the CDF values switch from 0.6 to 0.5 )

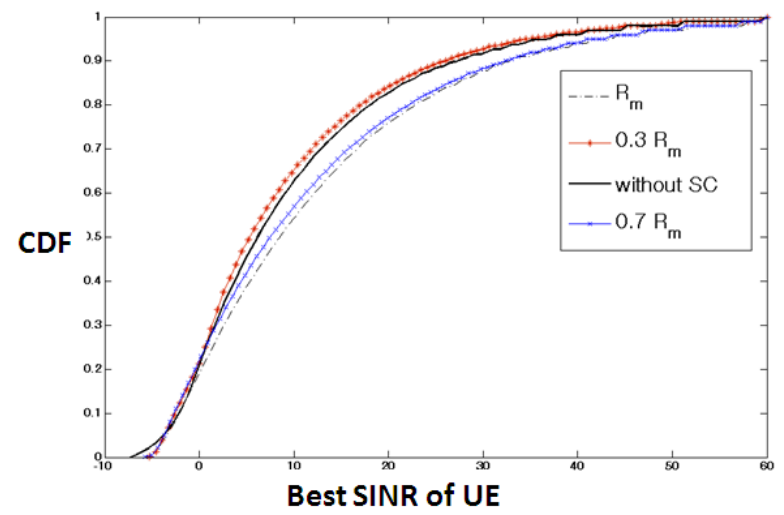

Fig. 4. CDF of the best SINR for different distances between MBS and SBS, and without small cell SC (black curve).

\section{Impact on SINR distribution in the zone covered by SBS}

This previous result is interesting since it allows to analyze the global impact of adding SBS on a MBS network. However, it cannot allow to analyze the local impact of SBS since the whole area covered by the MBS and SBS is taken into account, and not only the area covered by the SBS. Therefore, it seems interesting to analyze also the specific zone covered by added SBS. In this aim, for a heterogeneous network composed of MBS and SBS, we establish the CDF of the SINR of UEs located in the zone covered by a SBS, considering this last one as the best serving BS. Then, we compare it to the CDF of the SINR of UEs located at the same place, by considering a network only composed of MBS, i.e. when the SBS are not added (see figure 5). The figure 5 is drawn for a location of SBS at a distance $d=0.9 R_{m}$ from the MBS. The CDF are calculated by using expression (9) of the SINR (i.e. network without SBS, black curve) and expression (15) (i.e. network with MBS and SBS, red dotted curve). Figure 5 shows that adding SBS results in a very high improvement of the SINR, in the local zone it covers. Indeed, we first establish that, without SBS, the SINR range is between -4 and $5 \mathrm{~dB}$ in that zone. With SBS, the SINR range is between -4 and $30 \mathrm{~dB}$. Therefore, the coverage of the system is improved. For the same reason, the reachable QoS of UEs covered by $\mathrm{SBS}$, in terms of throughput and outage probability, is also highly improved by adding a SBS. For example, for a SINR of $-2.5 \mathrm{~dB}$, the outage reaches about $35 \%$ without SBS (i.e. UE connected to MBS) and decreases until $10 \%$ with SBS. Moreover, the maximum reachable SINR is about $5 \mathrm{~dB}$ (without SBS) and about $30 \mathrm{~dB}$ (with SBS). The analysis developed allows to quantify precisely the improvement in 
terms of QoS, performance and coverage by adding SBS in a macro cell network.

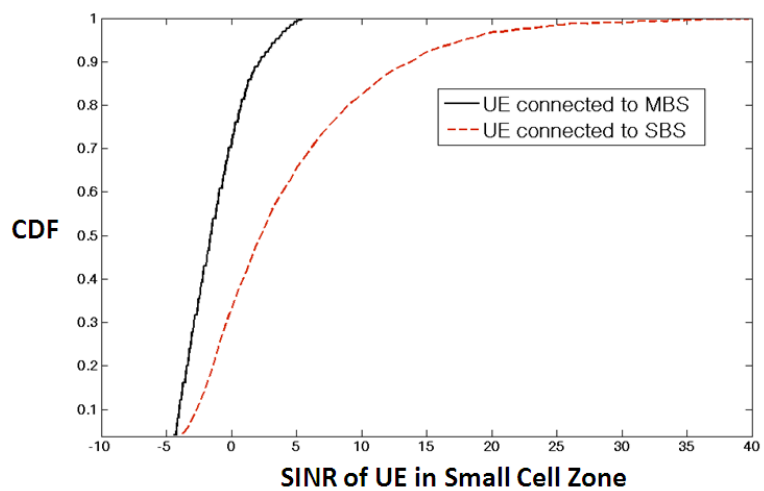

Fig. 5. CDF of the SINR in the zone covered by SBS, in the presence of SBS (red dotted line) and without SBS in the same zone (black continuous line).

\section{Impact of the SBS transmit power}

We investigate the impact of the SBS transmit power $P_{s}$ as we determined in expression of $\Omega_{m}$ (16) that only the ratio $\frac{P_{s}}{P_{m}}$ plays a role on the SINR (and thus the throughput) as expressed in (18) and (19). Moreover the transmitting power $P_{m}$ of MBS is fixed as a network constraint. Figure 6 shows the CDF of the throughput in the case of a deployment of SBS at the edge of macro cells, for transmitting powers $P_{s}$ of SBS of $0 \mathrm{dBm}$ and $20 \mathrm{dBm}$ and in the case without small cells $P_{s}=-\mathrm{Inf} \mathrm{dBm}$. It can be observed that, until an outage probability of $10 \%$, the CDF of the throughput is not impacted by the transmitting power of small cells. For higher values, we observe an increase of the outage probability when the transmitting power decreases. These curves also mean that the throughput performance of the system is improved when the transmitting power of small cells increases. For example, a probability of $50 \%$ is observed to reach $25 \mathrm{Mbits} / \mathrm{s}$ with $P_{s}=$ $20 \mathrm{dBm}, 20 \mathrm{Mbits} / \mathrm{s}$ with $P_{s}=0 \mathrm{dBm}$ and $18 \mathrm{Mbits} / \mathrm{s}$ without small cells.

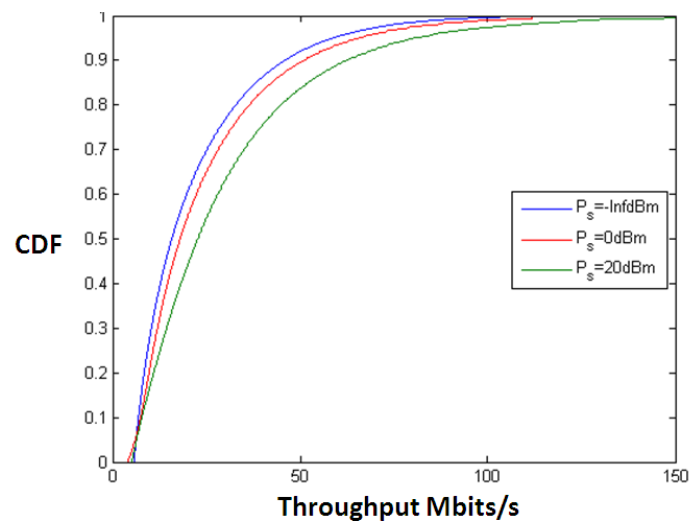

Fig. 6. CDF of the mean throughput.

\section{Conclusion}

In this paper, we analyzed and quantified in a simple way, the impact of adding small base stations in a network composed of macro base stations on the performance and the quality of service of the system. We considered an analytical approach for modeling networks composed of macro and small base stations. This approach allows to establish closed form expressions for the SINR of a user at any distance from its best server, which can be either a macro base station or a small base station. These expressions particularly enable to calculate in a simple way the performance and user QoS, and to analyze the coverage of heterogeneous networks. We analyzed the critical impact of small base stations locations. We showed that adding small base stations can locally improve the SINR, especially when they are located at the edges of the macro cells. In this case, the outage neither increases nor decreases. However, when the small cells are too close of the macro BS, the performance globally decreases. The analysis will be improved, in future works, by considering the shadowing impact.

\section{ACKNOWLEDGMENT}

The authors thank William Diego for his contribution to the work presented in this paper. This work was performed for the EU FP7 HARP project and France ANR NetLearn project.

\section{REFERENCES}

[1] S. Kaiser, Spatial Transmit Diversity Techniques for Broadband OFDM Systems, Proc. of Globecom, 2000.

[2] B. Badic, T. OFarrell, P. Loskot and J. He, Energy Efficient Radio Access Architectures for Green Radio: Large versus Small Cell Size Deployment, Proc. of VTC Fall 2009.

[3] M. Le Treust, H. Tembine, S. Lasaulce and M. Debbah, Coverage games in small cells networks, Proc. of Future Network and Mobile Summit 2010 Conference, IIMC International Information Management Corporation, 2010.

[4] T.-L. Sheu and C.-N. Lin, Adaptive Channel Preemption Model for SmallCell Embedded Large-Cellular Networks, Proc. of Wireless Communication Mobile Computing, 2011.

[5] J.-M. Kelif, W. Diego and S. Senecal, Impact of Transmitting Power on Femto Cells Performance and Coverage in Heterogeneous Wireless Networks, Proc. of WCNC, 2012.

[6] A. Damnjanovic, J. Montojo, Y. Wei, T. Ji, T. Luo, M.Vajapeyam, T. Yoo, O. Song and D. Malladi, A Survey on 3GPP Heterogeneous Networks, IEEE Wireless Communications, June 2011.

[7] D. Lopez-Perez, I. Guvenc, G. De La Roche, M. Kountouris, T. Q. S. Quek and J. Zhang, Enhanced Intercell Interference Coordination Challenges in Heterogeneous Networks, IEEE Wireless Communications, June 2011.

[8] J.-M. Kelif, M. Coupechoux and P. Godlewski, Spatial Outage Probability for Cellular Networks, Proc. of GLOBECOM, 2007.

[9] 3GPP TSG-RAN1\#48, 12th-16th February, 2007, St. Louis, MI, USA. R1-070674 (Appendix A).

[10] 3GPP TSG-RAN1 WG1, LTE Downlink Performance, Conference Call, Apr 24th 2007. R1-071978.

[11] P. Jacquet, Geometry of information propagation in massively dense ad hoc networks, Proc. of ACM MobiHoc, 2004.

[12] S. Toumpis and L. Tassiulas, Packetostatics Deployment of Massively Dense Sensor Networks as an Electrostatics Problem, Proc. of INFOCOM, 2005.

[13] J-M. Kelif, M. Coupechoux and P. Godlewski, On the Dimensioning of Cellular OFDMA Networks, Physical Communication Journal, Ref : PHYCOM118, online October 2011, DOI : 10.1016/j.phycom.2011.09.008. 